\title{
A DISTRIBUTIVE LATTICE CONNECTED WITH ARITHMETIC PROGRESSIONS OF LENGTH THREE
}

\author{
FU LIU AND RICHARD P. STANLEY
}

\begin{abstract}
Let $\mathcal{T}$ be a collection of 3 -element subsets $S$ of $\{1, \ldots, n\}$ with the property that if $i<j<k$ and $a<b<c$ are two 3-element subsets in $S$, then there exists an integer sequence $x_{1}<x_{2}<\cdots<$ $x_{n}$ such that $x_{i}, x_{j}, x_{k}$ and $x_{a}, x_{b}, x_{c}$ are arithmetic progressions. We determine the number of such collections $\mathcal{T}$ and the number of them of maximum size. These results confirm two conjectures of Noam Elkies.
\end{abstract}

\section{INTRODUCTION}

This paper has its origins in a problem contributed by Ron Graham to the Numberplay subblog of the New York Times Wordplay blog [1]. Graham asked whether it is always possible to two-color a set of eight integers such that there is no monochromatic three-term arithmetic progression. A proof was found by Noam Elkies. Let $\left(\begin{array}{c}{[n]} \\ 3\end{array}\right)$ denote the set of all three-element subsets of $[n]=\{1,2, \ldots, n\}$. Define two such subsets, say $i<j<k$ and $a<b<c$, to be consistent if there exist integers $x_{1}<x_{2}<\cdots<x_{n}$ for which both $x_{i}, x_{j}, x_{k}$ and $x_{a}, x_{b}, x_{c}$ are arithmetic progressions. For instance, $1<2<3$ and $1<2<4$ are obviously not consistent.

Let us call a collection $S$ of three-element subsets of integers valid if any two elements of $S$ are consistent. For instance, the valid subsets of $\left(\begin{array}{c}{[4]} \\ 3\end{array}\right)$ are

$$
\emptyset\{123\}\{124\}\{134\}\{234\}\{123,134\}\{123,234\}\{124,234\},
$$

so eight in all.

Elkies needed to generate all valid subsets of $\left(\begin{array}{c}{[8]} \\ 3\end{array}\right)$. Define $f(n)$ to be the number of valid subsets of $\left(\begin{array}{c}{[n]} \\ 3\end{array}\right)$, so $f(4)=8$ as noted above.

Date: July 2, 2018.

2010 Mathematics Subject Classification. 05A15.

Key words and phrases. consistent set, distributive lattice, semistandard Young tableau.

Fu Liu is partially supported by a grant from the Simons Foundation \#245939 and by NSF grant DMS-1265702.

Richard Stanley is partially supported by NSF grant DMS-1068625. 
Elkies needed to work with the case $n=8$, but he first computed that for $n \leq 7$ there are exactly $2^{\left(\begin{array}{c}n-1 \\ 2\end{array}\right)}$ such subsets, leading to the obvious conjecture that this formula holds for all $n \geq 1$. Elkies then verified this formula for $n=8$, using the list of valid subsets to solve Graham's problem. He then checked that $f(n)=2^{\left(\begin{array}{c}n-1 \\ 2\end{array}\right)}$ for $n=9$ and $n=10$. In Theorem 2.12 we show that indeed $f(n)=2^{\left(\begin{array}{c}n-1 \\ 2\end{array}\right)}$ for all $n \geq 1$.

Let $\sigma(n)$ be the size (number of elements) of the largest valid subset of $\left(\begin{array}{c}{[n]} \\ 3\end{array}\right)$. Elkies showed that

$$
\sigma(n)=\left\{\begin{aligned}
m(m-1), & n=2 m \\
m^{2}, & n=2 m+1 .
\end{aligned}\right.
$$

Let $g(n)$ be the number of valid subsets of $\left(\begin{array}{c}{[n]} \\ 3\end{array}\right)$ of maximal size $\sigma(n)$. Equation (1.1) shows that $\sigma(4)=2$ and $g(4)=3$. Elkies also conjectured (stated slightly differently) that

$$
g(n)=\left\{\begin{aligned}
2^{(m-1)(m-2)}\left(2^{m}-1\right), & n=2 m \\
2^{m(m-1)}, & n=2 m+1 .
\end{aligned}\right.
$$

We prove this conjecture in Section 5 .

The basic idea behind our two proofs is the following. After the Numberplay posting appeared, some further discussion continued on the domino email forum [2]. In particular, David desJardins observed that distinct triples $i<j<k$ and $i^{\prime}<j^{\prime}<k^{\prime}$ are inconsistent if and only if either

$$
i \leq i^{\prime}, j \geq j^{\prime}, k \leq k^{\prime}
$$

or

$$
i \geq i^{\prime}, j \leq j^{\prime}, k \geq k^{\prime} .
$$

(The proof is straightforward though somewhat tedious.) Jim Propp then defined a partial ordering $P_{n}$ on certain elements of $[n] \times[n] \times[n]$ such that the valid subsets of $\left(\begin{array}{c}{[n]} \\ 3\end{array}\right)$ are just the antichains of $P_{n}$. Since the antichains of a poset $P$ are just the maximal elements of order ideals of $P$, we get that $f(n)=\# L_{n}$, where $L_{n}:=J\left(P_{n}\right)$ denotes the (distributive) lattice of order ideals of $P_{n}$. One can also define a coordinate-wise partial ordering $M_{n}$ on the set of semistandard Young tableaux (SSYT) of shape $\delta_{n-1}:=(n-2, n-3, \ldots, 1)$ and largest part at most $n-1$. We show that $L_{n} \cong M_{n}$ by observing that both are distributive lattices and then showing that their posets of join-irreducibles are isomorphic. See [3, Thm. 3.4.1] for the relevant result on distributive lattices. It is an immediate consequence of standard results about SSYT that $\# M_{n}=2^{\left(\begin{array}{c}n-1 \\ 2\end{array}\right)}$, so the conjecture on $f(n)$ follows. The proof 
for $g(n)$ is more complicated. Let $K_{n}$ be the subset of $M_{n}$ corresponding to maximum size antichains of $P_{n}$ with respect to the isomorphism $L_{n} \rightarrow M_{n}$. By a result of Dilworth, $K_{n}$ is a sublattice of $M_{n}$ and is therefore distributive. We then determine the join-irreducibles of $K_{n}$. They are closely related to the join-irreducibles of $M_{1+\lfloor n / 2\rfloor}$, from which we are able to compute $g(n)=\# K_{n}$.

\section{The NUMBer OF VALID SUBSETS}

We assume the reader is familiar with basic definitions and results on posets and tableaux presented in [3, Chapter 3] and [4, Chapter 7]. Recall that for any graded poset $P$, its rank-generating function is

$$
F(P, q)=\sum_{x \in P} q^{\operatorname{rank}(x)}
$$

In this paper, we write a tableau $T$ using "English notation," so the longest row is at the top. Write $T_{a, b}=c$ to mean that the $(a, b)$-entry of $T$ is equal to $c$.

On April 17, 2013, Jim Propp posted on the Domino Forum [2] the following statement.

I don't know if this reformulation is helpful, but pairwise consistent sets are in bijection with antichains in the subposet of $[n] \times[n] \times[n]$ containing all the $(i, j, k)$ 's that satisfy $i+j<n+1<j+k$.

$([n] \times[n] \times[n]$ is the set $\{(i, j, k): 1 \leq i, j, k \leq n\}$, ordered so that $(i, j, k) \leq\left(i^{\prime}, j^{\prime}, k^{\prime}\right)$ iff $i \leq i^{\prime}$ and $j \leq j^{\prime}$ and $k \leq k^{\prime}$.)

To see the bijection, just map $(i, j, k)$ to $(i, n+1-j, k)$.

Propp's statement follows easily from the observation of David desJardins mentioned in the previous section.

Denote Propp's poset by $P_{n}$. The order ideals of $P_{n}$ form a distributive lattice $L_{n}=J\left(P_{n}\right)$ under inclusion [3, §3.4]. There is a simple bijection [3, end of $\S 3.1$ ] between the order ideals and antichains of a finite poset. Further, under this bijection, the size of an antichain of a poset $P$ is exactly the number of elements covered by the corresponding order ideal in $J(P)$. Hence,

(2.1) $f(n)=\# L_{n}$, the number of elements of $L_{n}$;

(2.2) $\sigma(n)=\max \left(\right.$ number of elements covered by $\left.x: x \in L_{n}\right)$;

(2.3) $g(n)=$ the number of $x \in L_{n}$ such that $x$ covers $\sigma(n)$ elements.

Recall from Section 1 that $M_{n}$ is the poset of all SSYT (semistandard Young tableaux) of shape $\delta_{n-1}=(n-2, n-3, \ldots, 1)$ and largest part 


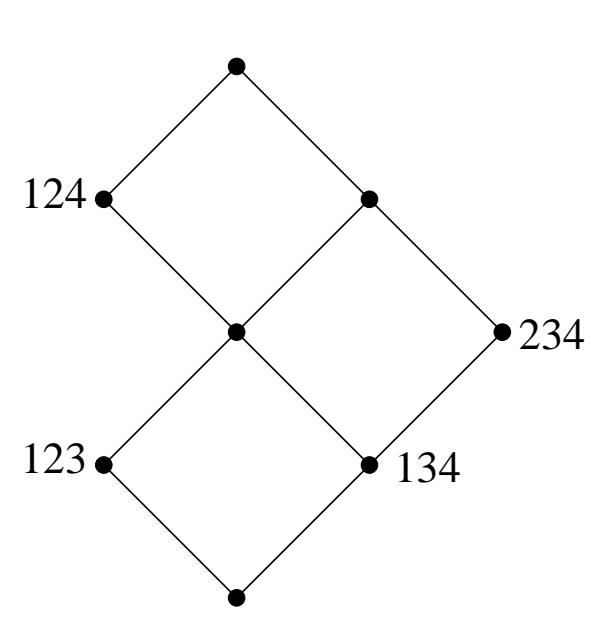

$L_{4}$

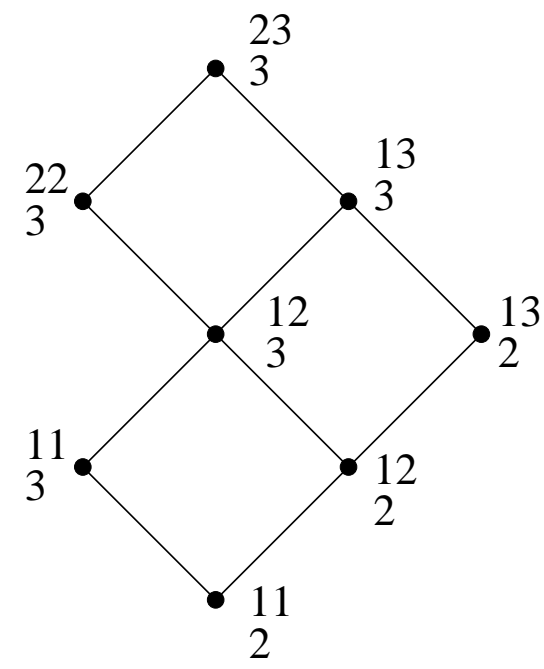

$M_{4}$

Figure 1. The distributive lattices $L_{4}$ and $M_{4}$

at most $n-1$, ordered componentwise. For $n \geq 2$ the poset $M_{n}$ is a distributive lattice, where join is entrywise maximum and meet is entrywise minimum, since either of the operations of maximum and minimum on the integers distributes over the other. We remark that $M_{1}$ is an empty set, which is neither interesting nor a distributive lattice. Hence throughout this paper, we assume $n \geq 2$. Let $Q_{n}$ denote the poset of join-irreducibles of $M_{n}$, so $M_{n}=J\left(Q_{n}\right)$.

Theorem 2.1. For $n \geq 2$,

$$
L_{n} \cong M_{n}
$$

We will show that $P_{n} \cong Q_{n}$ in Proposition 2.11. Hence by the fundamental theorem for finite distributive lattices [3. Thm. 3.4], $L_{n} \cong$ $M_{n}$. Theorem 2.1 follows. See Figure 1 for the lattices $L_{4}$ and $M_{4}$. We have labelled the join-irreducibles of $L_{4}$ by the corresponding elements of $P_{4}$. One can also confirm $f(4)=8, \sigma(4)=2$ and $g(4)=3$ by applying (2.1), (2.2), and (2.3) to the figure.

One main application of Theorem 2.1 is that we are able to describe $f(n), \sigma(n)$ and $g(n)$ as statistics related to the distributive lattice $M_{n}$. We say that an entry $c$ of a tableau $T \in M_{n}$ is reducible if by replacing $c$ with $c-1$ in $T$ we obtain another tableau in $M_{n}$.

Note 2.2. The $(a, b)$-entry of a SSYT $T$ is reducible if and only if

$$
T_{a, b}-T_{a, b-1} \geq 1 \quad \text { and } \quad T_{a, b}-T_{a-1, b} \geq 2,
$$


where by convention we let for all $a, b$,

$$
T_{a, 0}:=a \quad \text { and } \quad T_{0, b}:=0
$$

(although they are not real entries in $T$ ).

Corollary 2.3. For $n \geq 2$,

(a) $f(n)=\# M_{n}=\# J\left(Q_{n}\right)$.

(b) $\sigma(n)$ is the maximum number of reducible entries in $T$, for $T \in$ $M_{n}$.

(c) $g(n)$ is the number of tableaux in $M_{n}$ that have the maximum number $\sigma(n)$ of reducible entries.

Proof. It follows directly from Theorem 2.1, equations (2.1), (2.2) and (2.3), and the observation that for any $T \in M_{n}$, the number of elements covered by $T$ is the same as the number of reducible entries of $T$.

In order to prove $P_{n} \cong Q_{n}$, we first need to describe the poset $Q_{n}$ of join-irreducibles of $M_{n}$.

Definition 2.4. For any tableau $T$ with integer entries, we define $\operatorname{Add}(T ; a, b, k)$ to be the tableau obtained from $T$ by adding $k$ to each $\left(a^{\prime}, b^{\prime}\right)$-entry of $T$ with $\left(a^{\prime}, b^{\prime}\right) \geq(a, b)$.

Let $\mathcal{T}_{n-1}^{0}$ be the minimal element of $M_{n}$, so $\mathcal{T}_{n-1}^{0}$ is the tableau of shape $\delta_{n-1}$ whose $(a, b)$-entry is $a$.

Proposition 2.5. Let $\Phi_{n}$ be the set $\left\{(a, b, k) \in \mathbb{P}^{3} \mid 1 \leq k \leq b \leq\right.$ $n-1-a\}$ with the partial ordering

$$
(a, b, k) \leq_{\Phi_{n}}\left(a^{\prime}, b^{\prime}, k^{\prime}\right) \text { if } a \geq a^{\prime}, b \geq b^{\prime}, k \leq k^{\prime} .
$$

Then for any $(a, b, k) \in \Phi_{n}$, the tableau $\operatorname{Add}\left(\mathcal{T}_{n-1}^{0} ; a, b, k\right)$ is a joinirreducible of $M_{n}$. Moreover, all join-irreducible elements of $M_{n}$ are obtained in this way.

Furthermore, the map

$$
\psi:(a, b, k) \mapsto \operatorname{Add}\left(\mathcal{T}_{n-1}^{0} ; a, b, k\right)
$$

induces a poset isomorphism from $\Phi_{n}$ to $Q_{n}$.

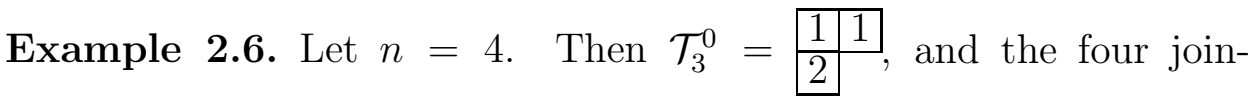
irreducibles of $M_{4}$ are:

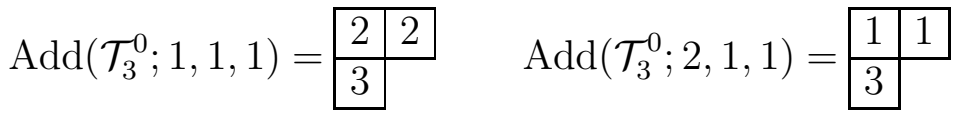

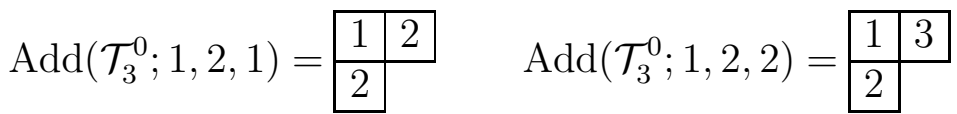

We need several preliminary results before proving Proposition 2.5. 
Lemma 2.7. Suppose $T$ is an $S S Y T$ and $T_{a, b}$ is an entry of $T$. If

$$
T_{a, b}-T_{a-1, b}>T_{a, b-1}-T_{a-1, b-1},
$$

then $T_{a, b}$ is a reducible entry.

Proof. We clearly have $T_{a, b}-T_{a-1, b} \geq 2$. Thus it is enough to show $T_{a, b}-T_{a, b-1} \geq 1$, which follows from $T_{a, b}-T_{a, b-1}>T_{a-1, b}-T_{a-1, b-1} \geq$ 0 .

Corollary 2.8. Suppose $T$ is an $S S Y T$ and $T_{a, b}$ is an entry of $T$. If

$$
T_{a, b}-T_{a-1, b}<T_{a, b-1}-T_{a-1, b-1},
$$

then there is a reducible entry in the a-th row of $T$.

Proof. (2.5) implies that $T_{a, b-1}-T_{a-1, b-1} \geq 2>1=T_{a, 0}-T_{a-1,0}$. Therefore there exists $1 \leq b^{\prime} \leq b-1$ such that

$$
T_{a, b^{\prime}}-T_{a-1, b^{\prime}}>T_{a, b^{\prime}-1}-T_{a-1, b^{\prime}-1} .
$$

Then the conclusion follows from Lemma 2.7.

Corollary 2.9. Suppose $T$ is an SSYT of shape $\lambda$. Then the following are equivalent.

(i) $T_{a_{0}, b_{0}}$ is the only reducible entry of $T$.

(ii) For any pair of indices $(a, b)$ such that $T_{a, b}$ is an entry of $T$, we have the following:

(a) $T_{a, b}-T_{a-1, b}=1$ for any $(a, b)$ satisfying $a \neq a_{0}$ or else satisfying $a=a_{0}$ and $b<b_{0}$;

(b) $T_{a, b}-T_{a-1, b} \geq 2$ for $(a, b)=\left(a_{0}, b_{0}\right)$;

(c) $T_{a, b}-T_{a-1, b}=T_{a, b-1}-T_{a-1, b-1}$ for any $(a, b)$ satisfying $a=a_{0}$ and $b>b_{0}$.

(iii) $T=\operatorname{Add}\left(\mathcal{T}^{0} ; a_{0}, b_{0}, k\right)$ for some $k \geq 1$, where $\mathcal{T}^{0}$ is the minimal SSYT of shape $\lambda$.

Proof. It is straightforward to verify that (ii) and (iii) are equivalent and that (iii) implies (i). We will show (i) implies (ii). Assuming (i), by Lemma 2.7 and Corollary 2.8, we have

$$
T_{a, b}-T_{a-1, b}=T_{a, b-1}-T_{a-1, b-1}, \text { for all }(a, b) \text { with } a \neq a_{0} .
$$

Note that

$$
T_{a, 0}-T_{a-1,0}=a-(a-1)=1, \quad \text { for all } a .
$$

We have $T_{a, b}-T_{a-1, b}=1$ when $a \neq a_{0}$. Since $T_{0, b}=0$ for any $b$, it follows that $T_{a, b}=a$ for any $a<a_{0}$. In particular,

$$
T_{a_{0}-1, b}=a_{0}-1, \quad \text { for all } b \text {. }
$$


Since the entries in the ath row are weakly increasing, we must have

$$
T_{a_{0}, b}-T_{a_{0}-1, b} \geq T_{a_{0}, b-1}-T_{a_{0}-1, b-1}, \quad \text { for all } b .
$$

Thus it follows from Lemma 2.7 that the equality of (2.7) holds when $b \neq b_{0}$ and inequality holds when $b=b_{0}$. Finally, it follows from (2.6) that condition (ii)(a) holds for $a=a_{0}$ and $b<b_{0}$ and thus condition (b) holds, completing the proof.

Proof of Proposition [2.5. We first verify the first part of the conclusion of the proposition, which is equivalent to say that $\psi$ gives a bijection from $\Phi_{n}$ to $Q_{n}$. Because of Corollary 2.9, it is sufficient to show that given $a+b \leq n-1$ (which implies that $(a, b)$ is an entry in a tableau of shape $\delta_{n-1}$ ), we have $k \leq b$ if and only if all the entries in $\operatorname{Add}\left(\mathcal{T}_{n-1}^{0} ; a, b, k\right)$ are at most $n-1$. However, note that the entries in $\mathcal{T}_{n-1}^{0}$ are less than $n-1$ and the largest entry in $\operatorname{Add}\left(\mathcal{T}_{n-1}^{0} ; a, b, k\right)$ that is different from $\mathcal{T}_{n-1}^{0}$ is the last entry in the $b$ th column of $\operatorname{Add}\left(\mathcal{T}_{n-1}^{0} ; a, b, k\right)$ :

$$
\operatorname{Add}\left(\mathcal{T}_{n-1}^{0} ; a, b, k\right)_{n-1-b, b}=n-1-b+k .
$$

Therefore each entry in $\operatorname{Add}\left(\mathcal{T}_{n-1}^{0} ; a, b, k\right)$ is at most $n-1$ if and only if $n-1-b+k \leq n-1$, which is equivalent to $k \leq b$. Thus the map $(a, b, k) \mapsto \operatorname{Add}\left(\mathcal{T}_{n-1}^{0} ; a, b, k\right)$ induces a bijection from $\Phi_{n}$ to $Q_{n}$.

It is easy to see that $\operatorname{Add}\left(\mathcal{T}_{n-1}^{0} ; a, b, k\right) \leq \operatorname{Add}\left(\mathcal{T}_{n-1}^{0} ; a^{\prime}, b^{\prime}, k^{\prime}\right)$ if and only if $a \geq a^{\prime}, b \geq b^{\prime}, k \leq k^{\prime}$. Hence we get an isomorphism, as desired.

We have the following corollary to Proposition 2.5 which will be used later.

Corollary 2.10. For $n \geq 2$,

$$
\# Q_{n+1}-\# Q_{n}=\left(\begin{array}{l}
n \\
2
\end{array}\right)
$$

Proof.

$$
\# Q_{n}=\sum_{k=1}^{n-2} \sum_{b=k}^{n-2}(n-1-b)=\sum_{k=1}^{n-2}\left(\begin{array}{c}
n-k \\
2
\end{array}\right)=\sum_{\alpha=1}^{n-2}\left(\begin{array}{c}
\alpha+1 \\
2
\end{array}\right) .
$$

Since we've shown that $Q_{n} \cong \Phi_{n}$ in Proposition 2.5, we establish that $P_{n} \cong Q_{n}$ by showing $P_{n} \cong \Phi_{n}$.

Proposition 2.11. Let $\Phi_{n}$ be the poset defined in Proposition 2.5. Define a map $\varphi: \Phi_{n} \rightarrow P_{n}$ by

$$
\varphi(a, b, k)=(k, n-b, n+1-a) .
$$


Then $\varphi$ is an isomorphism of posets.

Therefore, $P_{n} \cong \Phi_{n} \cong Q_{n}$.

Proof. This is just a straightforward verification. First we check that $\varphi(a, b, k) \in P_{n}$. We need to show that

$$
\begin{gathered}
1 \leq k \leq n, \quad 1 \leq n-b \leq n, \quad 1 \leq n+1-a \leq n \\
k+n-b<n+1<2 n+1-a-b .
\end{gathered}
$$

These inequalities are immediate from $(a, b, k) \in \Phi_{n}$, i.e., $1 \leq k \leq$ $b \leq n-1-a$ and $a, b, k \in \mathbb{P}$. We can then check that $\varphi^{-1}(i, j, \ell)=$ $(n+1-\ell, n-j, i)$. Hence $\varphi$ is a bijection $\Phi_{n} \rightarrow P_{n}$.

It remains to show that $\varphi$ is a poset isomorphism. However, one checks directly that $(a, b, k) \leq_{\Phi_{n}}\left(a^{\prime}, b^{\prime}, k^{\prime}\right)$ if and only if $k \leq k^{\prime}, n-b \leq$ $n-b^{\prime}, n+1-a \leq n+1-a^{\prime}$, i.e., $\varphi(a, b, k) \leq_{P_{n}} \varphi\left(a^{\prime}, b^{\prime}, k^{\prime}\right)$.

Therefore, as we discussed before, Theorem 2.1 follows from the above proposition.

Theorem 2.12. For any $n \geq 2$, the rank-generating function of $M_{n}$ is given by

$$
\begin{aligned}
F\left(M_{n}, q\right) & =(1+q)^{n-2}\left(1+q^{2}\right)^{n-3} \cdots\left(1+q^{n-2}\right) \\
& =\prod_{i=1}^{n-2}\left(1+q^{i}\right)^{n-1-i}
\end{aligned}
$$

where $F\left(M_{2}, q\right)=1$. Hence we have

$$
f(n)=2^{\left(\begin{array}{c}
n-1 \\
2
\end{array}\right)} .
$$

Proof. We compute $F\left(M_{n}, q\right)$ using standard results from the theory of symmetric functions. The rank of an element (SSYT) in $M_{n}$ is the sum of its entries minus $\left(\begin{array}{l}n \\ 3\end{array}\right)$. Denote the rank-generating function of $M_{n}$ by $F\left(M_{n}, q\right)$. If $T$ is an SSYT with $m_{i}$ entries equal to $i$, write $x^{T}=$ $x_{1}^{m_{1}} x_{2}^{m_{2}} \ldots$. The Schur function $s_{\delta_{n-2}}\left(x_{1}, \ldots, x_{n-1}\right)$ may be defined (see [4, Def. 7.10.1]) as

$$
s_{\delta_{n-2}}\left(x_{1}, \ldots, x_{n-1}\right)=\sum_{T} x^{T}
$$

where $T$ ranges over all SSYT of shape $\delta_{n-2}$ and largest part at most $n-1$. Hence

$$
q^{\left(\begin{array}{l}
n \\
3
\end{array}\right)} F\left(M_{n}, q\right)=s_{\delta_{n-2}}\left(q, \ldots, q^{n-1}\right) .
$$

Now we have [4, Exer. 7.30(a)]

$$
s_{\delta_{n-2}}\left(x_{1}, \ldots, x_{n-1}\right)=\prod_{1 \leq i<j \leq n-1}\left(x_{i}+x_{j}\right) .
$$


From this equation it is immediate that

$$
F\left(M_{n}, q\right)=(1+q)^{n-2}\left(1+q^{2}\right)^{n-3} \cdots\left(1+q^{n-2}\right) .
$$

Setting $q=1$ gives $f(n)=\# L_{n}=\# M_{n}=2^{\left(\begin{array}{c}n-1 \\ 2\end{array}\right)}$, completing the proof.

Note. Rather than using the special formula (2.11) we could have used the hook-content formula [4, Thm. 7.21.2] for $s_{\lambda}\left(1, q, \ldots, q^{m-1}\right)$, valid for any $\lambda$ and $m$. (Note that if $\lambda$ is a partition of $N$, then $q^{N} s_{\lambda}\left(1, q, \ldots, q^{m-1}\right)=s_{\lambda}\left(q, q^{2}, \ldots, q^{m}\right)$.) Equation (2.11) can be translated into some enumerative property of valid subsets of $\left(\begin{array}{c}{[n]} \\ 3\end{array}\right)$, but it seems rather contrived.

\section{VAlid SUBSETS OF MAXIMUM SIZE}

In the rest of the paper, we will prove Elkies' conjecture on the formula (1.3) for $g(n)$ as well as provide another proof for his formula (1.2) for $\sigma(n)$. Recall that in Corollary 2.3 we give alternative definitions for $\sigma(n)$ and $g(n)$ in terms of $M_{n}$. We find it is convenient to use the following obvious lemma to describe tableaux in $M_{n}$ using inequalities.

Lemma 3.1. Suppose $T$ is a tableau of shape $\delta_{n-1}$ with integer entries. Then $T \in M_{n}$ if and only if the entries of $T$ satisfy the following conditions:

(a) $T_{1,1} \geq 1$.

(b) $T_{a, b}-T_{a, b-1} \geq 0$, for any $2 \leq b \leq n-2$, $1 \leq a \leq n-1-b$ (weakly increasing on rows)

(c) $T_{a, b}-T_{a-1, b} \geq 1$, for any $2 \leq a \leq n-2,1 \leq b \leq n-1-a$ (strictly increasing on columns)

(d) $T_{n-1-b, b} \leq n-1$, for any $1 \leq b \leq n-2$.

Remark 3.2. For convenience, we sometimes abbreviate conditions (a) (c) of Lemma 3.1 as: for all $1 \leq b \leq n-2,1 \leq a \leq n-1-b$,

$$
T_{a, b}-T_{a, b-1} \geq 0 \quad \text { and } \quad T_{a, b}-T_{a-1, b} \geq 1, \quad,
$$

with the convention $T_{a, 0}=a$ and $T_{0, b}=0$.

Since $\sigma(n)$ is the maximum possible number of reducible entries in a tableau in $M_{n}$, we first give an upper bound for the number of reducible entries in $T \in M_{n}$.

Lemma 3.3. Let $T \in M_{n}$. Then for any $1 \leq b \leq n-2$,

\# reducible entries in the bth column of $T \leq \min (b, n-1-b)$. 
Therefore,

$$
\text { \# reducible entries in } T \leq \sum_{b=1}^{n-2} \min (b, n-1-b) \text {. }
$$

Proof. First, the number of reducible entries in the bth column is at most the number of entries in the $b$ th column, which is $n-1-b$.

By Lemma 3.1 (d), the last entry $T_{n-1-b, b}$ in the $b$ th column satisfies

$$
n-1 \geq T_{n-1-b, b}=\sum_{a=1}^{n-1-b}\left(T_{a, b}-T_{a-1, b}\right) .
$$

Hence by Remark 3.2 and Note 2.2 ,

$$
\begin{aligned}
n-1 \geq & \#(\text { irreducible entries in the } b \text { th column of } T)+ \\
& 2 \times \#(\text { reducible entries in the } b \text { th column of } T) \\
= & \#(\text { entries in the } b \text { th column of } T)+ \\
& \#(\text { reducible entries in the } b \text { th column of } T) \\
= & n-1-b+\#(\text { reducible entries in the } b \text { th column of } T) .
\end{aligned}
$$

Then we conclude that the number of reducible entries in the $b$ th column is at most $b$.

Definition 3.4. Let $K_{n}$ be the coordinate-wise partial ordering on the set of all the tableaux in $M_{n}$ that have $\sum_{b=1}^{n-2} \min (b, n-1-b)$ reducible entries. (Thus $K_{n}$ is a subposet of $M_{n}$.)

Remark 3.5. By Lemma 3.3, we see that $\sigma(n)$ is at most

$$
\sum_{b=1}^{n-2} \min (b, n-1-b)=\left\{\begin{array}{l}
1+2+\cdots+(m-1)+(m-1)+\cdots+2+1 \\
=m(m-1), \quad \text { if } n=2 m ; \\
1+2+\cdots+(m-1)+m+(m-1)+\cdots+2+1 \\
=m^{2}, \quad \text { if } n=2 m+1 .
\end{array}\right.
$$

One only needs show that $K_{n}$ is nonempty to confirm Elkies' formula (1.2) for $\sigma(n)$. Although one can easily directly construct a tableau that is in $K_{n}$, we choose to start by analyzing the properties of tableaux in $K_{n}$ and give a proof for the nonemptyness of $K_{n}$ indirectly in the next section. The benefit of doing this is that the arguments are useful for figuring out the cardinality of $K_{n}$, which gives the value of $g(n)$. 
Properties of tableaux in $K_{n}$. We have the following immediate consequence of Lemma 3.3 and its proof.

Lemma 3.6. Suppose $T \in M_{n}$. Then $T \in K_{n}$ if and only if the following two conditions are satisfied.

(a) For any $1 \leq b \leq \frac{1}{2}(n-1)$, the number of reducible entries in the bth column of $T$ is $b$.

(b) For any $\frac{1}{2}(n-1)<b \leq n-2$, all the entries in the bth column of $T$ are reducible.

While condition (b) of the above lemma is enough for us to determine how to create the right half entries of tableaux in $K_{n}$, we will discuss explicit conditions for the left half entries as a consequence of Lemma 3.6)(a) in several corollaries below.

Corollary 3.7. Suppose $T \in K_{n}$. Then for any $1 \leq b \leq \frac{1}{2}(n-1)$,

(a) the last entry $T_{n-1-b, b}$ in the bth column of $T$ is $n-1$;

(b) for any $1 \leq a \leq n-1-b$, the entry $T_{a, b}$ is reducible if and only if $T_{a, b}-T_{a-1, b}=2$, and $T_{a, b}$ is irreducible if and only if $T_{a, b}-T_{a-1, b}=1$.

Therefore,

(c) among all the $n-1-b$ entries $T_{a, b}$ in the bth column of $T$, there are $b$ entries satisfying $T_{a, b}-T_{a-1, b}=2$, and the remaining $n-1-2 b$ entries satisfying $T_{a, b}-T_{a-1, b}=1$.

Proof. By Lemma 3.6(a) the number of reducible entries in the $b$ th column of $T$ is $b$. However, by the proof of Lemma 3.3. one sees that this only happens when the equalities in both (3.2) and (3.1) hold. Therefore (a) and (b) follow, and then (c) follows.

Corollary 3.8. Suppose $T \in K_{n}$. Then for any $1 \leq b \leq \frac{1}{2}(n-1)-1$ and $1 \leq a \leq n-1-b$ (so both $T_{a, b}$ and $T_{a-1, b+1}$ are on the left half of T), we have

$$
T_{a, b}-T_{a-1, b+1} \leq 1
$$

Proof. Assume to the contrary that

$$
T_{a, b}-T_{a-1, b+1} \geq 2 .
$$

We claim that $a+b<n-1$. If $a=1$, then $1+b \leq \frac{1}{2}(n-1)<n-1$; if $a>1$, then by Corollary 3.7(a) the condition $a+b=n-1$ implies $T_{a, b}=n-1=T_{a-1, b+1}$, which is impossible. Thus $a+b<n-1$, and so $a+(b+1) \leq n-1$. Hence $T$ has an $(a, b+1)$-entry. Then by Corollary $3.7(b)$,

$$
T_{a, b+1}-T_{a-1, b+1} \leq 2
$$


Comparing with our assumption (3.4), we conclude that $T_{a, b+1} \leq T_{a, b}$. However, since $T$ is a SSYT, one has to have

$$
T_{a, b+1}=T_{a, b} .
$$

Thus both equalities in (3.4) and (3.5) hold. In particular, we get $T_{a, b+1}-T_{a-1, b+1}=2$. Now using Corollary [3.7(b) we conclude that $T_{a, b+1}$ is reducible. However, by Note 2.2 this implies that $T_{a, b+1}-T_{a, b} \geq$ 1 , which contradicts equation (3.6).

It turns out that (3.3) is an important property. Since it is related to the difference of two consecutive northeast-southwest diagonal entries, we often refer to it as "the diagonal property." Below we give an easy but useful lemma for using this property.

Lemma 3.9. Suppose $T$ is a tableau (of some shape) filled with integer entries. Assume $b \geq 1$ and $a \geq 0$ and the $(a, b),(a+1, b)$ and $(a, b+1)$ entries of $T$ satisfy

$$
T_{a+1, b}-T_{a, b+1} \leq 1 \quad \text { and } \quad T_{a+1, b}-T_{a, b} \geq 1 .
$$

Then

$$
T_{a, b} \leq T_{a, b+1},
$$

where the equality holds if and only if $T_{a+1, b}=T_{a, b}+1=T_{a, b+1}+1$.

This lemma says that the diagonal property together with the property of strictly increasing on columns implies the property of weakly increasing on rows.

Proof. We combine the two inequalities in (3.7):

$$
T_{a, b}+1 \leq T_{a+1, b} \leq T_{a, b+1}+1 .
$$

Then the conclusion follows.

Corollary 3.10. Suppose $T \in K_{n}$. Then

$$
T_{a, b}=a, \quad \text { for all } 1 \leq b \leq \frac{1}{2}(n-1), 0 \leq a \leq \frac{1}{2}(n-1)-b .
$$

Therefore for any $1 \leq b \leq \frac{1}{2}(n-1)$ and $1 \leq a \leq \frac{1}{2}(n-1)-b$, the entry $T_{a, b}$ is irreducible. Hence the first $\left\lfloor\frac{1}{2}(n-1)\right\rfloor-b$ entries (not counting $\left.T_{0, b}\right)$ in the bth column are irreducible for any $1 \leq b \leq\left\lfloor\frac{1}{2}(n-1)\right\rfloor$.

Proof. We prove (3.8) by induction on a noting that the indicies in (3.8) can be described as

$$
0 \leq a \leq \frac{1}{2}(n-1)-1,1 \leq b \leq \frac{1}{2}(n-1)-a .
$$

The base case when $a=0$ clearly holds since $T_{0, b}=0$ by our convention. Suppose (3.8) holds for $a=a_{0}$ for some $0 \leq a_{0} \leq \frac{1}{2}(n-1)-2$. We 
want to show $T_{a, b}=a$, for $a=a_{0}+1$ and any $1 \leq b \leq \frac{1}{2}(n-1)-a$. One checks that $a$ and $b$ satisfy

$$
1 \leq b \leq \frac{1}{2}(n-1)-1 \quad \text { and } \quad 1 \leq a \leq \frac{1}{2}(n-1)-b \leq n-1-b .
$$

Hence by Corollary 3.8, we have the diagonal property

$$
T_{a, b}-T_{a-1, b+1} \leq 1 \text {. }
$$

Meanwhile, since $T$ is an SSYT, we have

$$
T_{a, b}-T_{a-1, b} \geq 1 \text {. }
$$

However, by the induction hypothesis,

$$
T_{a-1, b}=a_{0}=T_{a-1, b+1} .
$$

It follows from Lemma 3.9 that

$$
T_{a, b}=T_{a-1, b}+1=(a-1)+1=a .
$$

Hence (3.8) holds.

The second conclusion easily follows from (3.8) and Corollary 3.7(b).

We are now ready to state and prove the main result of this section.

Proposition 3.11. Suppose $T$ is a tableau of shape $\delta_{n-1}$ filled with integer entries. Then $T \in K_{n}$ if and only if the following conditions are satisfied.

(a) For any $1 \leq b \leq\left\lfloor\frac{1}{2}(n-1)\right\rfloor$,

(i) for any $1 \leq a \leq\left\lfloor\frac{1}{2}(n-1)\right\rfloor-b$, we have $T_{a, b}=a$;

(ii) among the $\lfloor n / 2\rfloor$ remaining values of $a$, viz., $\left\lfloor\frac{1}{2}(n-1)\right\rfloor-$ $b+1 \leq a \leq n-1-b$, we have that $b$ of them satisfy $T_{a, b}-T_{a-1, b}=2$, and the remaining $\lfloor n / 2\rfloor-b$ of them satisfy $T_{a, b}-T_{a-1, b}=1$.

(b) For any $1 \leq b \leq\left\lfloor\frac{1}{2}(n-1)\right\rfloor-1$ and $\left\lfloor\frac{1}{2}(n-1)\right\rfloor-b+1 \leq a \leq$ $n-1-b$, we have the diagonal property $T_{a, b}-T_{a-1, b+1} \leq 1$.

(c) For any $\left\lfloor\frac{1}{2}(n-1)\right\rfloor+1 \leq b \leq n-2$ and any $1 \leq a \leq n-1-b$, we have $T_{a, b} \leq n-1, T_{a, b}-T_{a-1, b} \geq 2$ and $T_{a, b}-T_{a, b-1} \geq 1$.

Proof. Suppose $T \in K_{n}$. It follows from Lemma 3.6, Corollaries 3.7 and 3.8, and Note 2.2 that (a)-(c) hold.

Now suppose (a)-(c) hold. We first show that $T \in M_{n}$ by verifying that the conditions in Lemma 3.1 are satisfied. It is clear that conditions (a) and (c) of Lemma 3.1 hold. One sees that (a) implies that $T_{n-1-b, b}=n-1$ for any $1 \leq b \leq\left\lfloor\frac{1}{2}(n-1)\right\rfloor$, which together with (c), implies condition (d) of Lemma 3.1 . 
Thus we only need to show that for any $2 \leq b \leq n-2,1 \leq a \leq$ $n-1-b$,

$$
T_{a, b}-T_{a, b-1} \geq 0 \text {. }
$$

However, we already know that (3.9) holds for $\left\lfloor\frac{1}{2}(n-1)\right\rfloor+1 \leq b \leq n-2$ by condition (c), and holds for $2 \leq b \leq\left\lfloor\frac{1}{2}(n-1)\right\rfloor$ and $1 \leq a \leq$ $\left\lfloor\frac{1}{2}(n-1)\right\rfloor-b$ by condition (a)(i). Thus we assume $2 \leq b \leq\left\lfloor\frac{1}{2}(n-1)\right\rfloor$ and $\left\lfloor\frac{1}{2}(n-1)\right\rfloor-b+1 \leq a \leq n-1-b$. Hence by $(\mathrm{b})$, we have the diagonal property

$$
T_{a+1, b-1}-T_{a, b} \leq 1 .
$$

Then (3.9) follows from Lemma 3.9, so we conclude that $T \in M_{n}$.

Now it suffices to verify conditions (a) and (b) of Lemma 3.6 to conclude $T \in K_{n}$. Lemma 3.6(b) clearly follows from (c). For condition (a) of Lemma 3.6, because of Lemma 3.3, it is enough to check that for any $1 \leq b \leq\left\lfloor\frac{1}{2}(n-1)\right\rfloor$ and $\left\lfloor\frac{1}{2}(n-1)\right\rfloor-b+1 \leq a \leq n-1-b$, if $T_{a, b}-T_{a-1, b}=2$, then $T_{a, b}$ is reducible. However, by Note 2.2, it is sufficient to prove

$$
T_{a, b}-T_{a-1, b}=2 \quad \Longrightarrow \quad T_{a, b}-T_{a, b-1} \geq 1 .
$$

If $b=1$, since $T_{a, b-1}=a=T_{a-1, b-1}+1 \leq T_{a-1, b}+1$, we immediately have $T_{a, b}-T_{a, b-1} \geq 1$. If $b>1$ and $a=\left\lfloor\frac{1}{2}(n-1)\right\rfloor-b+1$, it follows from condition (a) (i) that $T_{a, b-1}-T_{a-1, b}=a-(a-1)=1$. Thus (3.10) holds. Hence we assume $2 \leq b \leq\left\lfloor\frac{1}{2}(n-1)\right\rfloor$ and $\left\lfloor\frac{1}{2}(n-1)\right\rfloor-b+2 \leq$ $a \leq n-1-b$. Applying (b) again, we get $T_{a, b-1}-T_{a-1, b} \leq 1$. Then (3.10) follows.

\section{TWO SIDES}

Since the characterization of the left half and right half of tableaux in $K_{n}$ stated in Proposition 3.11 are quite different, it is natural to split each $T \in K_{n}$ into two halves and investigate them separately.

Let $\delta_{n-1}^{\mathcal{L}}$ be the shape that is the left half of $\delta_{n-1}$ including the middle column if there is one. In other words, $\delta_{n-1}^{\mathcal{L}}$ is the conjugate of $(n-2, n-3, \ldots,\lfloor n / 2\rfloor)$. Note that the shape of the right half of $\delta_{n-1}$ excluding the middle column is $\delta_{\lfloor n / 2\rfloor}$.

Definition 4.1. Let $K_{n}^{\mathcal{L}}$ be the set of all the tableaux of shape $\delta_{n-1}^{\mathcal{L}}$ with integer entries satisfying conditions (a) and (b) of Proposition 3.11. For $c=1$ or 2 , let $K_{n}^{\mathcal{L}, c}$ be the subset of $K_{n}^{\mathcal{L}}$ consisting all tableaux whose $\left(1,\left\lfloor\frac{1}{2}(n-1)\right\rfloor\right)$ )-entry is $c$. (Note that the $\left(1,\left\lfloor\frac{1}{2}(n-1)\right\rfloor\right)$-entry is the last entry in the first row of any tableau in $K_{n}^{\mathcal{L}}$, which has to be either 1 or 2 by condition (a) of Proposition 3.11.) 
For $c=1$ or 2 , let $K_{n}^{\mathcal{R}, c}$ be the set of all the tableaux of shape $\delta_{\lfloor n / 2\rfloor}$ satisfying the following conditions:

a) $T_{1,1} \geq c+1$.

b) $T_{a, b}-T_{a, b-1} \geq 1$ for any $2 \leq b \leq\lfloor n / 2\rfloor-1,1 \leq a \leq\lfloor n / 2\rfloor-b$.

c) $T_{a, b}-T_{a-1, b} \geq 2$, for any $2 \leq a \leq\lfloor n / 2\rfloor-1,1 \leq b \leq\lfloor n / 2\rfloor-a$,

d) $T_{\lfloor n / 2\rfloor-b, b} \leq n-1$, for any $1 \leq b \leq\lfloor n / 2\rfloor-1$.

We consider all the sets above as posets with the coordinate-wise partial ordering.

For any $T \in K_{n}$, we define $\operatorname{Split}(T)=\left(T^{\mathcal{L}}, T^{\mathcal{R}}\right)$, where $T^{L}$ of shape $\delta_{n-1}^{\mathcal{L}}$ is the left half including the middle column of $T$, and $T^{\mathcal{R}}$ of shape $\delta_{\lfloor n / 2\rfloor}$ is the right half excluding the middle column of $T$. It follows from Proposition 3.11 that $\left(T^{\mathcal{L}}, T^{\mathcal{R}}\right) \in K_{n}^{\mathcal{L}, c} \times K_{n}^{\mathcal{R}, c}$ for some $c=1$ or 2. Thus,

$$
\operatorname{Split}\left(K_{n}\right) \subseteq\left(K_{n}^{\mathcal{L}, 1} \times K_{n}^{\mathcal{R}, 1}\right) \bullet\left(K_{n}^{\mathcal{L}, 2} \times K_{n}^{\mathcal{R}, 2}\right) .
$$

The equality in the above equation actually holds.

Lemma 4.2. We have

$$
\operatorname{Split}\left(K_{n}\right)=\left(K_{n}^{\mathcal{L}, 1} \times K_{n}^{\mathcal{R}, 1}\right) \bullet\left(K_{n}^{\mathcal{L}, 2} \times K_{n}^{\mathcal{R}, 2}\right) .
$$

Therefore,

$$
K_{n} \cong\left(K_{n}^{\mathcal{L}, 1} \times K_{n}^{\mathcal{R}, 1}\right) \cup\left(K_{n}^{\mathcal{L}, 2} \times K_{n}^{\mathcal{R}, 2}\right) .
$$

Proof. We only need to show that for any $\left(T^{\mathcal{L}}, T^{\mathcal{R}}\right) \in K_{n}^{\mathcal{L}, c} \times K_{n}^{\mathcal{R}, c}$ for some $c=1$ or 2 , if $T$ is the tableau obtained by gluing $T^{\mathcal{L}}$ on the left side of $T^{\mathcal{R}}$, then we have $T \in K_{n}$. However, by Proposition 3.11 , it is enough to verify that

$$
T_{a, 1}^{\mathcal{R}}-T_{a,\left\lfloor\frac{1}{2}(n-1)\right\rfloor}^{\mathcal{L}} \geq 1, \quad \text { for all } a .
$$

It follows from conditions (a) and (c) of Definition 4.1 that $T_{a, 1}^{\mathcal{R}} \geq$ $c+1+2(a-1)$. Further, because $T^{\mathcal{L}}$ satisfies condition (a) of Proposition 3.11 and $T_{1,\left\lfloor\frac{1}{2}(n-1)\right\rfloor}^{\mathcal{L}}=c$, we conclude that $T_{a,\left\lfloor\frac{1}{2}(n-1)\right\rfloor}^{\mathcal{L}} \leq c+2(a-1)$. Therefore equation (4.1) follows.

The main goal of this section is to show

$$
K_{n}^{\mathcal{L}} \cong M_{\lfloor n / 2\rfloor+1}
$$

Definition 4.3. Let $A_{n}$ be the set of tableaux of shape $\delta_{n-1}^{\mathcal{L}}$ with integer entries satisfying condition (a) of Proposition 3.11,

Write $m=\left\lfloor\frac{1}{2}(n-1)\right\rfloor$. Let $A_{n}^{\prime}$ be the set of tableaux of rectangular shape

$$
(\underbrace{m, m, \ldots, m}_{\lfloor n / 2\rfloor})
$$


with entries 1 or 2 where the $b$ th column has $b$ copies of 2 's and $\lfloor n / 2\rfloor-b$ copies of 1's.

Let $A_{n}^{\prime \prime}$ be the set of tableaux of shape $\delta_{\lfloor n / 2\rfloor}$ with integer entries in $\{1,2, \ldots,\lfloor n / 2\rfloor\}$ where the entries in each column are strictly increasing.

Define $\theta_{1}: A_{n} \rightarrow A_{n}^{\prime}$ in the following way. For any $T \in A_{n}$, we do the following three operations on $T$.

(1) For any $1 \leq b \leq m$ and $1 \leq a \leq m-b$, we replace the number in the $(a, b)$-entry with $T_{a, b}-T_{a-1, b}$.

(2) Remove all the entries $T_{a, b}$ with $a+b \leq m$. (Note after this each column has $\lfloor n / 2\rfloor$ entries left.)

(3) Shift all the entries up to make a rectangular shape.

We call the resulting rectangular tableau $\theta_{1}(T)$.

Define $\theta_{2}: A_{n}^{\prime} \rightarrow A_{n}^{\prime \prime}$ in the following way. For any $T^{\prime} \in A_{n}^{\prime}$, we create $\theta_{2}\left(T^{\prime}\right)$ of shape $\theta_{\lfloor n / 2\rfloor}$ with entries

$$
\theta_{2}\left(T^{\prime}\right)_{a, b}:=\text { the row index of the } a \text { th } 1 \text { in column } b \text { of } T^{\prime} .
$$

Define $\theta=\theta_{2} \circ \theta_{1}: A_{n} \rightarrow A_{n}^{\prime \prime}$.

Example 4.4. Below are examples of the maps $\theta_{1}$ and $\theta_{2}$ for $n=6$ and $n=7$.
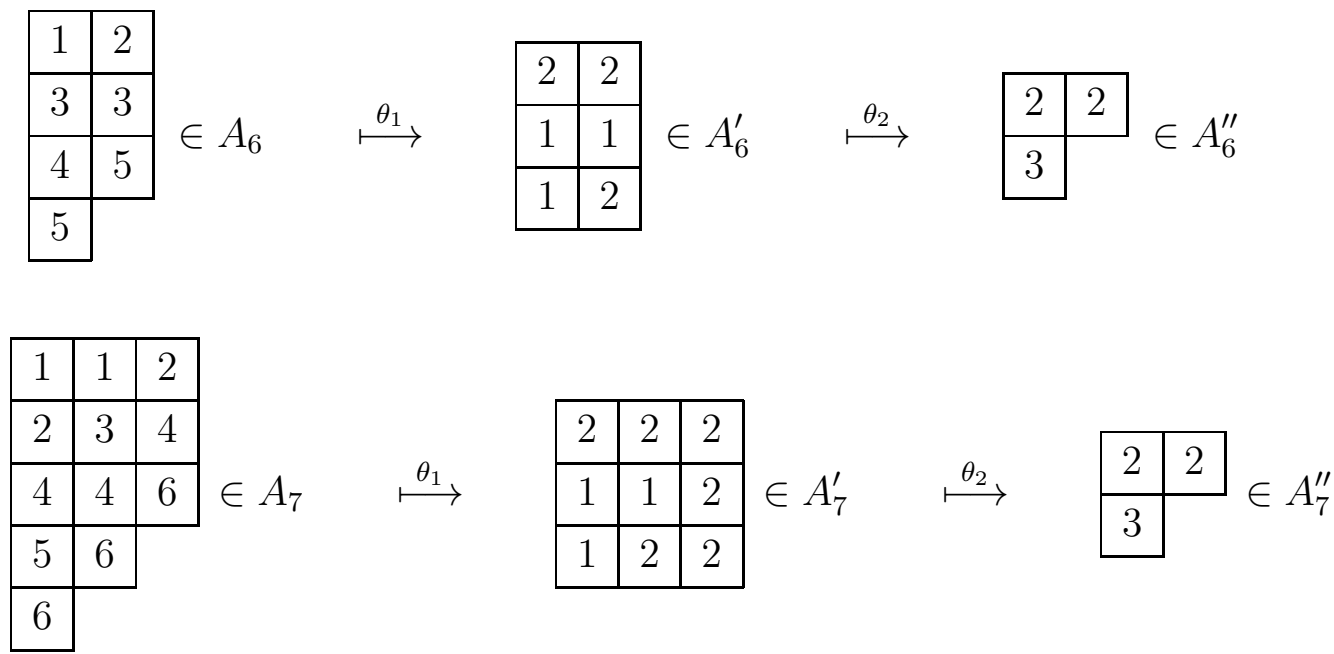

It is clear that $\theta_{1}$ is a bijection from $A_{n}$ to $A_{n}^{\prime}$ and $\theta_{2}$ is a bijection from $A_{n}^{\prime}$ to $A_{n}^{\prime \prime}$. Hence $\theta$ is a bijection from $A_{n}$ to $A_{n}^{\prime \prime}$. Below is the main result of this section.

Proposition 4.5. Consider both $A_{n}$ and $A_{n}^{\prime \prime}$ as posets with the coordinatewise partial ordering. Then $\theta$ is a poset isomorphism from $A_{n}$ to $A_{n}^{\prime \prime}$. 
Furthermore (noting that $K_{n}^{\mathcal{L}}$ is a subposet of $A_{n}$ ) the map $\theta$ induces a poset isomorphism from $K_{n}^{\mathcal{L}}$ to $M_{\lfloor n / 2\rfloor+1}$. Hence,

$$
K_{n}^{\mathcal{L}} \cong M_{\lfloor n / 2\rfloor+1}
$$

We will break the proof of Proposition 4.5 into several lemmas. For convenience, for any column $C$ with entries in $\{1,2\}$, we define

$$
\text { \#Ones }(C, i):=\text { the number of } 1 \text { 's in the first } i \text { entries of } C \text {, }
$$

and

$$
\operatorname{RI}(C, a):=\text { the row index of the } a \text { th } 1 \text { of } C .
$$

For example, if $C$ is the first column of the $3 \times 2$ tableau in $A_{6}^{\prime}$ appearing in Example 4.4, we have $\# \operatorname{Ones}(C, 1)=0, \# \operatorname{Ones}(C, 2)=1$, \#Ones $(C, 3)=2$.

We have the following obvious lemma on these two statistics.

Lemma 4.6. Suppose $C$ and $C^{\prime}$ are two columns of $\ell$ entries in $\{1,2\}$. Then the following two conditions are equivalent.

(i) For any $1 \leq i \leq \ell$, \#Ones $(C, i) \leq \# \operatorname{Ones}\left(C^{\prime}, i\right)$.

(ii) \#Ones $(C, \ell) \leq \#$ Ones $\left(C^{\prime}, \ell\right)$ and $\operatorname{RI}(C, a) \geq \operatorname{RI}\left(C^{\prime}, a\right)$ for any $1 \leq a \leq \# \operatorname{Ones}(C, \ell)$.

Proof. Suppose (i) holds. Clearly we have \#Ones $(C, \ell) \leq \# \operatorname{Ones}\left(C^{\prime}, \ell\right)$. Let $1 \leq a \leq \# \operatorname{Ones}(C, \ell)$ and $\operatorname{RI}(C, a)=i$. Then

$$
a=\# \operatorname{Ones}(C, i) \leq \# \operatorname{Ones}\left(C^{\prime}, i\right) .
$$

Therefore $\operatorname{RI}\left(C^{\prime}, a\right) \leq i$, so (ii) follows.

Suppose there exists $1 \leq i \leq \ell$ such that \#Ones $(C, i)>$ \#Ones $\left(C^{\prime}, i\right)$. Let $a=\#$ Ones $(C, i)$. Then $\operatorname{RI}(C, a) \leq i<\operatorname{RI}\left(C^{\prime}, a\right)$.

Note that with the definition of RI, we can rewrite (4.2) as

$$
\theta_{2}\left(T^{\prime}\right)_{a, b}:=\operatorname{RI}\left(\text { column } b \text { of } T^{\prime}, a\right) .
$$

Lemma 4.7. Suppose $T^{(1)}, T^{(2)} \in A_{n}$. Then the following conditions are equivalent.

(a) $T^{(1)} \leq T^{(2)}$.

(b) For any $1 \leq j \leq\left\lfloor\frac{1}{2}(n-1)\right\rfloor$ and $1 \leq i \leq\lfloor n / 2\rfloor$,

\#Ones $\left(\right.$ column $j$ of $\left.\theta_{1}\left(T^{(1)}\right), i\right) \geq \#$ Ones $\left(\right.$ column $j$ of $\left.\theta_{2}\left(T^{(2)}\right), i\right)$.

(c) $\theta\left(T^{(1)}\right) \leq \theta\left(T^{(2)}\right)$.

Proof. The equivalence between (b) and (c) follows directly from Lemma 4.6 . 
Write $m=\left\lfloor\frac{1}{2}(n-1)\right\rfloor$. For any $T \in A_{n}$, any $1 \leq j \leq m$ and $1 \leq i \leq\lfloor n / 2\rfloor$, we have

$$
\begin{aligned}
T_{m-j+i, j}-T_{m-j, j} & =\sum_{a=m-j+1}^{m-j+i}\left(T_{a, j}-T_{a-1, j}\right) \\
& =\sum_{k=1}^{i} \theta_{1}(T)_{k, j} \\
& =2 i-\# \text { Ones }\left(\operatorname{column} j \text { of } \theta_{1}(T), i\right) .
\end{aligned}
$$

Since $T$ satisfies condition (a)(i) of Proposition 3.11, we have $T_{m-j, j}=$ $m-j$. Thus,

$$
T_{m-j+i, j}=2 i+m-j-\# \text { Ones }\left(\operatorname{column} j \text { of } \theta_{1}(T), i\right) .
$$

Therefore (a) and (b) are equivalent.

One sees that the first conclusion of Proposition 4.5 follows from Lemma 4.7,

Lemma 4.8. Suppose $T \in A_{n}$. Then the following conditions are equivalent.

a) T satisfies condition (b) of Proposition 3.11.

b) For any $1 \leq j \leq\left\lfloor\frac{1}{2}(n-1)\right\rfloor-1$ and $1 \leq i \leq\lfloor n / 2\rfloor$,

\#Ones $\left(\right.$ column $j$ of $\left.\theta_{1}(T), i\right) \geq$ \#Ones $($ column $j+1$ of $\theta(T), i)$.

c) The entries are weakly increasing in each row of $\theta(T)$.

Proof. The proof is similar to that of Lemma 4.7. The equivalence between (b) and (c) follows from Lemma 4.6, and the equivalence between (a) and (b) follows from (4.3).

Lemma 4.8 implies that $T \in K_{n}^{\mathcal{L}}$ if and only if $\theta(T) \in M_{\lfloor n / 2\rfloor+1}$. Hence the second part of Proposition 4.5 follows. Below we give a result on the minimal element of $K_{n}^{\mathcal{L}}$ that will be used in the next section.

Lemma 4.9. The last entry in the first row of the unique minimal element of $K_{n}^{\mathcal{L}}$ is 1 if $n$ is even and is 2 if $n$ is odd.

Proof. Note that the minimal element of $M_{\lfloor n / 2\rfloor+1}$ is the tableau whose $(a, b)$-entry is $a$. It is easy to determine the minimal element in $K_{n}^{\mathcal{L}}$ which is in bijection with this minimal element under the map $\theta$, and check that it satisfies the condition described by the lemma.

In the rest of this section, we discuss some results on $K_{n}^{\mathcal{L}, c}$ and $K_{n}^{\mathcal{R}, c}$. 
Lemma 4.10. For $m \geq 2$,

$$
K_{2 m}^{\mathcal{L}, 1} \cong M_{m}
$$

Proof. Suppose $T \in K_{2 m}^{\mathcal{L}}$ and $T^{\prime}=\theta(T)$.

One checks that $T \in K_{2 m}^{\mathcal{L}, 1}$ if and only if the only 1 in the last column of $\theta_{1}(T)$ is in the first row, which is equivalent to the fact that the last entry in the first row of $T^{\prime}=\theta(T)$ is 1 .

However, since $T^{\prime}$ is an SSYT, the last entry in the first row of $T^{\prime}$ is 1 if and only if the entries in the first row of $T^{\prime}$ are all $1 \mathrm{~s}$. There is a natural bijection between tableaux in $M_{m+1}$ whose first row is all 1s and tableaux in $M_{m}$ : taking a tableau in the former set, we remove the first row and subtract 1 from each of the remaining entries, and obtain a tableau in $M_{m}$. It is clear that the composition of $\theta$ and this bijection gives a poset isomorphism from $K_{2 m}^{\mathcal{L}, 1}$ to $M_{m}$.

Definition 4.11. For $m \geq 1$, let $\mathcal{T}_{m}$ be the tableau of shape $\delta_{m}$ with entries

$$
\left(\mathcal{T}_{m}\right)_{a, b}=2 a+b, \quad \text { for all } 1 \leq b \leq m-1,1 \leq a \leq m-b,
$$

and $\mathcal{T}_{m}^{\prime}$ the tableau of shape $\delta_{m}$ with entries

$$
\left(\mathcal{T}_{m}^{\prime}\right)_{a, b}=2 a+b-1, \quad \text { for all } 1 \leq b \leq m-1,1 \leq a \leq m-b,
$$

Example 4.12. Let $m=3$. Then

$$
\mathcal{T}_{3}=\begin{array}{|l|l|}
\hline 3 & 4 \\
\hline 5 &
\end{array} \quad \text { and } \quad \mathcal{T}_{3}^{\prime}=\begin{array}{|l|l|}
\hline 2 & 3 \\
\hline 4 &
\end{array}
$$

Lemma 4.13. For any $n \geq 2$, we have

$$
K_{n}^{\mathcal{R}, 1} \supseteq K_{n}^{\mathcal{R}, 2} .
$$

Further, $\mathcal{T}_{\lfloor n / 2\rfloor}$ is the unique minimal element of $K_{n}^{\mathcal{R}, 2}$ and $\mathcal{T}_{\lfloor n / 2\rfloor}^{\prime}$ is the unique minimal element of $K_{n}^{\mathcal{R}, 1}$.

Hence, the unique minimal element of $K_{n}^{\mathcal{R}, 2}$ is greater than the unique minimal element of $K_{n}^{\mathcal{R}, 1}$.

Proof. (4.4) follows directly from the defintion of $K_{n}^{\mathcal{R}, 1}$ and $K_{n}^{\mathcal{R}, 2}$.

It is clear that if $\mathcal{T}_{\lfloor n / 2\rfloor} \in K_{n}^{\mathcal{R}, 2}$, it has to be the unique minimal element of $K_{n}^{\mathcal{R}, 2}$. Hence we only need to show that $\mathcal{T}_{\lfloor n / 2\rfloor} \in K_{n}^{\mathcal{R}, 2}$, which can be proved by verifying conditions (a)-(d) of Definition 4.1. We can similarly prove the statement on $\mathcal{T}_{\lfloor n / 2\rfloor}^{\prime}$.

Lemma 4.14. Let $m \geq 1$.
a) $K_{2 m}^{\mathcal{R}, 1} \cong M_{m+1}$
b) $K_{2 m+1}^{\mathcal{R}, 2} \cong M_{m+1}$. 
Proof. We first prove (a). For any $T \in M_{m+1}$, we define $\phi(T)$ to be the tableau of shape $\delta_{m}$ with entries

$$
\phi(T)_{a, b}=T_{a, b}+a+b-1 .
$$

Comparing Lemma 3.1 and Definition 4.1, one sees that $\phi(T) \in K_{2 m+1}^{\mathcal{R}, 2}$. Hence $\phi: M_{m+1} \rightarrow K_{2 m}^{\mathcal{R}, 1}$. It is easy to define the inverse map of $\phi$ and verify that $\phi$ is an poset isomorphism.

b) can be proved similarly by defining a map $\phi^{\prime}: M_{m+1} \rightarrow K_{2 m+1}^{\mathcal{R}, 2}$ where

$$
\phi^{\prime}(T)_{a, b}=T_{a, b}+a+b
$$

We finish this section by concluding the nonemptyness of $K_{n}$, which leads to Elkie's formula for $\sigma(n)$ as we've discussed in Remark 3.5.

Corollary 4.15. For any $n \geq 2$, the poset $K_{n}$ is nonempty.

Proof. By Proposition 4.5 and Lemma 4.13, the sets $K_{n}^{\mathcal{L}}, K_{n}^{\mathcal{R}, 1}$ and $K_{n}^{\mathcal{R}, 2}$ are all nonempty. Therefore the conclusion follows from Lemma 4.2 ,

Corollary 4.16 (Elkies). For $m \geq 1$,

$$
\sigma(n)=\sum_{b=1}^{n-2} \min (b, n-1-b)= \begin{cases}m(m-1), & \text { if } n=2 m \\ m^{2}, & \text { if } n=2 m+1 .\end{cases}
$$

\section{JOIN-IRREDUCIBLES OF $K_{n}$}

In the last section we confirmed Elkies' formula for $\sigma(n)$. As a consequence, the value $g(n)$ is the cardinality of $K_{n}: g(n)=\# K_{n}$. In this section, we will determine rank-generating function of $K_{n}$, which leads to a formula for $\# K_{n}$ by discussing the structure of the poset of join-irreducibles of $K_{n}$.

Let $U_{n}$ be the poset of the join-irreducibles of $K_{n}$. By a result of Dilworth (see Exercise 3.72(a) of [3]), $K_{n}$ is a distributive lattice. Hence by the fundamental theorem for finite distributive lattices, we have $K_{n}=J\left(U_{n}\right)$.

Suppose $T \in K_{n}$ and $\operatorname{Split}(T)=\left(T^{\mathcal{L}}, T^{\mathcal{R}}\right)$. Let $c$ be the last entry in the first row of $T^{\mathcal{L}}$. It is clear that $T$ is a join-irreducible if and only if one of the following two cases happens:

(1) $T^{\mathcal{L}}$ is a join-irreducible of $K_{n}^{\mathcal{L}}$, and $T^{\mathcal{R}}$ is the unique minimal element in $K_{n}^{\mathcal{R}, c}$.

(2) $T^{\mathcal{L}}$ is the unique minimal element in $K_{n}^{\mathcal{L}}$, and $T^{\mathcal{R}}$ is a joinirreducible of $K_{n}^{\mathcal{R}, c}$. 
We call $T \in K_{n}$ a left-join-irreducible if it fits into situation (1), and a right-join-irreducible if it fits into situation (2). Let $U_{n}^{\ell}\left(U_{n}^{r}\right.$, respectively) be the subposet of $U_{n}$ that consists of all the left-joinirreducibles (right-join-irreducibles, respectively). Further, for $i=1,2$ we let $U_{n}^{\ell, c}$ be the set of those $T$ that fit into situation (1) with $c=i$. (Thus $U_{n}^{\ell}$ is the disjoint union of $U_{n}^{\ell, 1}$ and $U_{n}^{\ell, 2}$.)

Note that for a given $n$, since the unique minimal element in $K_{n}^{\mathcal{L}}$ is fixed the number $c$ in situation (2) is fixed. Using Lemma 4.9, we can give a more explicit description of $U_{n}^{r}$ depending on the parity of $n$.

Lemma 5.1. Suppose $T \in K_{n}$ and $\operatorname{Split}(T)=\left(T^{\mathcal{L}}, T^{\mathcal{R}}\right)$.

(a) For even $n, T \in U_{n}^{r}$ if and only if $T^{\mathcal{L}}$ is the unique minimal element in $K_{n}^{\mathcal{L}}$ and $T^{\mathcal{R}}$ is a join-irreducible of $K_{n}^{\mathcal{R}, 1}$.

(b) For odd $n, T \in U_{n}^{r}$ if and only if $T^{\mathcal{L}}$ is the unique minimal element in $K_{n}^{\mathcal{L}}$ and $T^{\mathcal{R}}$ is a join-irreducible of $K_{n}^{\mathcal{R}, 2}$.

Recall that $Q_{n}$ is the poset of join-irreducibles of $M_{n}$.

Lemma 5.2. For $n \geq 2$, the following are true.

(a) $U_{n}^{\ell} \cong Q_{\lfloor n / 2\rfloor+1}$.

(b) $U_{n}^{\ell, 1} \cong$ the poset of join-irreducibles of $K_{n}^{\mathcal{L}, 1}$.

(c) $U_{n}^{r} \cong Q_{\lfloor n / 2\rfloor+1}$.

Proof. Suppose for $i=1,2$ we have $T^{(i)} \in U_{n}^{\ell}$ and $\operatorname{Split}\left(T^{(i)}\right)=$ $\left(T^{(i), \mathcal{L}}, T^{(i), \mathcal{R}}\right)$. By Lemma 4.13, we have $T^{(1)} \leq T^{(2)}$ if and only if $T^{(1), \mathcal{L}} \leq T^{(2), \mathcal{L}}$. Therefore $U_{n}^{\ell}$ is isomorphic to the poset of joinirreducibles of $K_{n}^{\mathcal{L}}$. Hence (a) follows from Proposition 4.5.

For any $T \in K_{n}^{\mathcal{L}, 1}$, because it does not cover any element in $K_{n}^{\mathcal{L}, 2}$ in the poset $K_{n}^{\mathcal{L}}$, the elements covered by $T$ in $K_{n}^{\mathcal{L}}$ are exactly the same as the elements covered by $T$ in $K_{n}^{\mathcal{L}, 1}$. Hence (b) follows from the fact that $U_{n}^{\ell}$ is isomorphic to the poset of join-irreducibles of $K_{n}^{\mathcal{L}}$.

By Lemma 5.1, $U_{n}^{r}$ is isomorphic to the poset of join-irreducibles of $K_{n}^{\mathcal{R}, 1}$ if $n$ is even and isomorphic to the poset of join-irreducibles of $K_{n}^{\mathcal{R}, 2}$ if $n$ is odd. It follows from Lemma 4.14 that $U_{n}^{r} \cong Q_{\lfloor n / 2\rfloor+1}$.

Lemma 5.3. Suppose $T^{\ell} \in U_{n}^{\ell}$ and $T^{r} \in U_{n}^{r}$ are left-join-irreducible and right-join-irredubcile of $K_{n}$, respectively. For $s=\ell, r$, let $\operatorname{Split}\left(T^{s}\right)=$ $\left(T^{s, \mathcal{L}}, T^{s, \mathcal{R}}\right)$ and $c^{s}$ the last entry in the first row of $T^{s, \mathcal{L}}$. Then the following are equivalent.

(i) $T^{\ell}$ and $T^{r}$ are comparable.

(ii) $T^{r}<T^{\ell}$.

(iii) $c^{r}=1, c^{\ell}=2$, and $T^{r, \mathcal{R}} \leq \mathcal{T}_{\lfloor n / 2\rfloor}$. (Recall that $\mathcal{T}_{\lfloor n / 2\rfloor}$ defined in Definition 4.11 is the unique minimal element of $K_{n}^{\mathcal{R}, 2}$.) 
Proof. Since $T^{r, \mathcal{L}}$ is the minimal element of $K_{n}^{\mathcal{L}}$, we clearly have $T^{r, \mathcal{L}}<$ $T^{\ell, \mathcal{L}}$. Therefore (i) and (ii) are equivalent. Furthermore, we have condition (ii) is equivalent to $T^{r, \mathcal{R}} \leq T^{\ell, \mathcal{R}}$.

Note that if $c^{\ell}=2, T^{\ell, \mathcal{R}}=\mathcal{T}_{\lfloor n / 2\rfloor}$. Hence (iii) implies (ii). Now we assume (ii) which implies $T^{r, \mathcal{R}} \leq T^{\ell, \mathcal{R}}$. If $c^{r} \geq c^{\ell}$, then by Lemma 4.13,

$$
\begin{aligned}
T^{r, \mathcal{R}} & >\text { the unique minimal element in } K_{n}^{\mathcal{R}, c^{r}} \\
& \geq \text { the unique minimal element in } K_{n}^{\mathcal{R}, c^{\ell}}=T^{\ell, \mathcal{L}} .
\end{aligned}
$$

Thus we must have $c^{r}=1$ and $c^{\ell}=2$. Then $T^{\ell, \mathcal{R}}=\mathcal{T}_{\lfloor n / 2\rfloor}$ and (iii) follows.

Because of condition (iii) in Lemma 5.3, it is natural for us to divide $U_{n}^{r}$ into two sets as well.

Definition 5.4. Let $U_{n}^{r, 2}$ be the subposet of $U_{n}^{r}$ that consists of all tableaux $T$ such that $T^{\mathcal{R}} \leq \mathcal{T}_{\lfloor n / 2\rfloor}$, where $\operatorname{Split}(T)=\left(T^{\mathcal{L}}, T^{\mathcal{R}}\right)$.

Let $U_{n}^{r, 1}$ be the set $U_{n}^{r} \backslash U_{n}^{r, 2}$ with the coordinate-wise partial ordering.

Note that in Lemma 5.3, $T^{r}$ is a right-join-irreducible and thus $c^{r}$ is the last entry in the first row of the minimal element of $K_{n}^{\mathcal{L}}$. Thus by Lemma 4.9, $c^{r}$ is always 1 for even $n$ and is always 2 for odd $n$. Applying Lemma 5.3 to odd cases and even cases separately, we have the following results.

Corollary 5.5. Suppose $T^{\ell} \in U_{n}^{\ell}$ and $T^{r} \in U_{n}^{r}$ are left-join-irreducibles and right-join-irredubciles of $K_{n}$, respectively.

(a) If $n$ is odd, then $T^{\ell}$ and $T^{r}$ are incomparable.

(b) Suppose $n$ is even. Let $\operatorname{Split}\left(T^{r}\right)=\left(T^{r, \mathcal{L}}, T^{r, \mathcal{R}}\right)$. Then the following are equivalent.

(i) $T^{\ell}$ and $T^{r}$ are comparable.

(ii) $T^{r}<T^{\ell}$.

(iii) $T^{\ell} \in U_{n}^{\ell, 2}$ and $T^{r} \in U_{n}^{r, 2}$.

We now have enough information to determine the rank-generating function of $K_{n}$ for odd $n$.

Theorem 5.6. Suppose $n=2 m+1$ for some $m \geq 1$. Then

$$
K_{n} \cong M_{m+1} \times M_{m+1} \cong J\left(Q_{m+1}+Q_{m+1}\right) .
$$

Therefore the rank-generating function of $K_{n}$ is given by

$$
F\left(K_{n}, q\right)=\left((1+q)^{m-1}\left(1+q^{2}\right)^{m-2} \cdots\left(1+q^{m-1}\right)\right)^{2},
$$

where $F\left(K_{3}, q\right)=1$. 
Proof. Corollary 5.5 implies that for odd $n$, we have as posets,

$$
U_{n}=U_{n}^{\ell}+U_{n}^{r}
$$

Then (5.1) follows from Lemma 5.2, Applying Theorem 2.12, we obtain (5.2).

Remark 5.7. Theorem 5.6 can be proved more directly without discussing the poset $U_{n}$ of join-irreducibles. One can argue that for odd $n$, the set $K_{n}^{\mathcal{L}, 1}$ is empty and thus $K_{n} \cong K_{n}^{\mathcal{L}, 2} \times K_{n}^{\mathcal{R}, 2}=K_{n}^{\mathcal{L}} \times K_{n}^{\mathcal{R}, 2}$. Then (5.1) follows from Proposition 4.5 and Lemma 4.14.

Substituting $q=1$ in (5.2) gives us the cardinality of $K_{n}$, which is the value of $g(n)$.

Corollary 5.8. Suppose $n=2 m+1$ for some $m \geq 1$. Then

$$
g(n)=\# K_{n}=2^{m(m-1)} \text {. }
$$

We focus on the case where $n$ is even for the rest of this section.

Lemma 5.9. For $m \geq 2$ we have $U_{2 m}^{r, 1} \cong Q_{m}$.

Proof. First, it's clear that

$$
U_{2 m}^{r, 1} \cong\left\{T \not \subset \mathcal{T}_{m} \mid T \text { is a join-irreducible of } K_{2 m}^{\mathcal{R}, 1}\right\},
$$

where $\mathcal{T}_{m}$ as defined in Definition 4.11 is the minimal element of $K_{2 m}^{\mathcal{R}, 2}$. Recall that in Proposition 2.5 we define a poset $\Phi_{n}$ and a poset isomorphism $\psi: \Phi_{n} \rightarrow Q_{n}$, and in the proof of Lemma 4.14 we define a poset isomorphism $\phi: M_{m+1} \rightarrow K_{2 m}^{\mathcal{R}, 1}$. Letting $n=m+1$ and taking the composition of $\psi$ and $\phi$, we obtain an isomorphism from $\Phi_{m+1}$ to the poset of join-irreducibles of $K_{2 m}^{\mathcal{R}, 1}$. Further, it is easy to see that

$$
\begin{aligned}
\phi(\psi((a, b, k)) & =\phi\left(\operatorname{Add}\left(\mathcal{T}_{m}^{0} ; a, b, k\right)\right) \\
& =\operatorname{Add}\left(\phi\left(\mathcal{T}_{m}^{0}\right) ; a, b, k\right) \\
& =\operatorname{Add}\left(\mathcal{T}_{m}^{\prime} ; a, b, k\right),
\end{aligned}
$$

where $\mathcal{T}_{m}^{\prime}$ as defined in Definition 4.11 is the minimal element of $K_{2 m}^{\mathcal{R}, 1}$. Comparing the definitions of $\mathcal{T}_{m}^{\prime}$ and $\mathcal{T}_{m}$, one sees that $\mathcal{T}_{m}=\operatorname{Add}\left(\mathcal{T}_{m}^{\prime} ; 1,1,1\right)$. Hence,

$$
\operatorname{Add}\left(\mathcal{T}_{m}^{\prime} ; a, b, k\right) \not \mathcal{T}_{m} \quad \Longleftrightarrow \quad k \geq 2 .
$$

Therefore the right-hand side of (5.3) is isomorphic to

$$
\begin{aligned}
\left\{(a, b, k) \in \Phi_{m+1} \mid k \geq 2\right\} & =\left\{(a, b, k) \in \mathbb{P}^{3} \mid 2 \leq k \leq b \leq m-a\right\} \\
& \cong\left\{\left(a, b^{\prime}, k^{\prime}\right) \in \mathbb{P}^{3} \mid 1 \leq k^{\prime} \leq b^{\prime} \leq m-1-a\right\} \\
& \quad\left(k^{\prime}=k-1, b^{\prime}=b-1\right) \\
& =\Phi_{m},
\end{aligned}
$$


which is isomorphic to $Q_{m}$ by Proposition 2.5 .

At this point, we have a good understanding of the structure of $U_{n}$ for even $n$. We summarize this in the following proposition.

Proposition 5.10. Suppose $n=2 m$ for $m \geq 2$. The poset $U_{n}$ of the join-irreducibles of $K_{n}$ can be divided into two disjoint sets $U_{n}^{\ell}$ and $U_{n}^{r}$, each of which are divided into two disjoint sets $U_{n}^{\ell}=U_{n}^{\ell, 1} \cup U_{n}^{\ell, 2}$ and $U_{n}^{r}=U_{n}^{r, 1} \cup U_{n}^{r, 2}$ such that they satsify the following conditions:

a) $U_{n}^{\ell} \cong U_{n}^{r} \cong Q_{m+1}$.

b) $U_{n}^{\ell, 1} \cong U_{n}^{r, 1} \cong Q_{m}$.

c) No element in $U_{n}^{\ell, 1}$ is comparable to any element in $U_{n}^{r}$.

d) No element in $U_{n}^{r, 1}$ is comparable to any element in $U_{n}^{\ell}$.

e) Each element of $U_{n}^{r, 2}$ is smaller than any element in $U_{n}^{\ell, 2}$.

Proof. (a) follows from Lemma 5.2(a,c), and (c)-(e) follow from Corollary 5.5(b). Finally, (b) follows from Lemma 5.2(b), Lemma 4.10, and Lemma 5.9.

Theorem 5.11. Suppose $n=2 m$ for some $m \geq 2$. Then the rankgenerating function of $K_{n}$ is given by

$$
\begin{aligned}
F\left(K_{n}, q\right)= & \left((1+q)^{m-2}\left(1+q^{2}\right)^{m-1} \cdots\left(1+q^{m-2}\right)\right)^{2} \\
& \cdot\left((1+q)\left(1+q^{2}\right) \cdots\left(1+q^{m-1}\right) \times\left(1+q^{\left(\begin{array}{c}
m \\
2
\end{array}\right)}\right)-q^{\left(\begin{array}{c}
m \\
2
\end{array}\right)}\right),
\end{aligned}
$$

where $F\left(K_{4}, q\right)=(1+q)^{2}-q=1+q+q^{2}$.

Proof. The part of $F\left(K_{n}, q\right)=F\left(J\left(U_{n}\right), q\right)$ which corresponds to order ideals that do not contain any element of $U_{n}^{\ell, 2}$ is

$$
\begin{aligned}
F\left(J\left(U_{n}^{r}+U_{n}^{\ell, 1}\right), q\right) & =F\left(J\left(U_{n}^{r}\right), q\right) F\left(J\left(U_{n}^{\ell, 1}\right), q\right) \\
& =F\left(M_{m+1}, q\right) F\left(M_{m}, q\right),
\end{aligned}
$$

and the part corresponding to order ideals that contain at least one element from $U_{n}^{\ell, 2}$ (and thus contain all the elements in $U_{n}^{r, 2}$ ) is

$$
\begin{aligned}
& \left(F\left(J\left(U_{n}^{\ell}\right), q\right)-F\left(J\left(U_{n}^{\ell, 1}\right), q\right)\right) \times q^{\# U_{n}^{r, 2}} \times F\left(J\left(U_{n}^{r, 1}\right), q\right) \\
= & \left(F\left(M_{m+1}, q\right)-F\left(M_{m}, q\right)\right) \times q^{\# Q_{m+1}-\# Q_{m}} \times F\left(M_{m}, q\right) .
\end{aligned}
$$

We obtain the formula for $F\left(K_{n}, q\right)$ by adding (5.4) and (5.5)), and then substituting from formulas (2.9) and (2.8).

Corollary 5.12. Suppose $n=2 m$ for some $m \geq 1$. Then

$$
g(n)=\# K_{n}=2^{(m-1)(m-2)}\left(2^{m}-1\right) \text {. }
$$


Proof. If $m=1$, one checks directly that $K_{n}$ contains one element. Hence, $g(2)=\# K_{2}=1=2^{0}\left(2^{1}-1\right)$.

For $m \geq 2$ the conclusion follows from substituting $q=1$ in the formula for $F\left(K_{n}, q\right)$ given in Theorem 5.11.

\section{REFERENCES}

[1] G. Antonick, The Improbable Life of Paul Erdös, New York Times Wordplay blog, March 25, 2013, http://wordplay.blogs.nytimes.com/2013/03/25/erdos.

[2] J. Propp (moderator), Domino forum, http://faculty.uml .edu/jpropp/about-domino.txt.

[3] R. P. Stanley, Enumerative Combinatorics, vol. 1, second ed., Cambridge Studies in Advanced Mathematics, vol. 49, Cambridge University Press, Cambridge, 2012.

[4] R. P. Stanley, Enumerative Combinatorics, vol. 2, Cambridge Studies in Advanced Mathematics, vol. 62, Cambridge University Press, Cambridge, 1999.

Fu Liu, Department of Mathematics, University of California, Davis, One Shields Avenue, Davis, CA 95616 USA.

E-mail address: fuliu@math.ucdavis.edu

Richard Stanley, Department of Mathematics, M.I.T., Cambridge, MA 02139 USA.

E-mail address: rstan@math.mit.edu 